\title{
INTERAÇÃO GENÓTIPO X AMBIENTE E ESTABILIDADE FENOTÍPICA DE CANA-DE-AÇÚCAR EM CICLO DE CANA DE ANO ( $\left.{ }^{1}\right)$
}

\author{
MARCELO DE ALMEIDA SILVA $(2 *)$
}

\begin{abstract}
RESUMO
A recomendação de variedades de cana-de-açúcar com desempenho superior em diversos ambientes é um desafio para melhoristas e fitotecnistas devido à presença de interação genótipo x ambiente. Pesquisas sobre estabilidade de genótipos de cana-de-açúcar no ciclo de cana de ano são escassas. Os objetivos deste trabalho foram avaliar a magnitude da interação genótipo x ambiente e determinar os genótipos estáveis no sistema de plantio cana de ano. Estabilidade fenotípica foi avaliada por meio de dois métodos, coeficiente de regressão linear e coeficiente de variação. Dez genótipos e duas variedades-padrão foram avaliados para altura de colmos, diâmetro de colmos, número de colmos por metro, pol\% cana e produtividade de cana e de açúcar em três locais em 2004 e 2005 . Houve interação genótipo x ambiente para diâmetro de colmos, número de colmos por metro, produtividade de colmos e de açúcar, observandose nos genótipos comportamento diferente em cada local. Número de colmos por metro foi o atributo biométrico mais afetado pela interação genótipo x ambiente. Os genótipos IAC87-3396, IAC91-1099 e IACSP94-4004 podem ser recomendados para plantio sob o sistema cana de ano nas regiões de Jaú, Piracicaba e São João da Boa Vista.
\end{abstract}

Palavras-chave: Saccharum sp., adaptação ambiental, atributos biométricos, produtividade, variedades.

\section{ABSTRACT \\ GENOTYPE X ENVIRONMENT INTERACTION AND PHENOTYPIC STABILITY IN SUGARCANE UNDER A TWELVE-MONTHS PLANTING CYCLE}

The recommendation of sugarcane varieties that show superior performance in different locations is a challenge for breeders and crop managers due to the response of genotypes across environments. Researches on the stability of sugarcane genotypes under a twelve-months planting cycle are scarce. The objectives of this work were to assess the magnitude of the genotype $x$ environment interaction and identify stable genotypes under this cycle. Phenotypic stability was estimated by two methods of analysis, linear regression coefficient and coefficient of variation. Ten genotypes and two control varieties were evaluated for stalk height, stalk diameter, stalk number per meter, pol\% cane and yield of cane and sugar in three locations during the years 2004 and 2005. The results showed that there was genotype $x$ environment interaction for stalk diameter, number of stalk and yield of cane and sugar, indicating that genotypes ranked differently at individual location. Number of stalk per meter was the biometric attribute more affected by the genotype $x$ environment interaction. The genotypes IAC87-3396, IAC91-1099 and IACSP94-4004 can be recommended for planting under a twelve months cycle at Jaú, Piracicaba and São João da Boa Vista regions of São Paulo State, Brazil.

Key words: Saccharum sp., environmental adaptability, biometric attributes, productivity, varieties.

$\left({ }^{1}\right)$ Recebido para publicação em 28 de dezembro de 2006 e aceito em 12 de julho de 2007.

$\left(^{2}\right)$ APTA Regional Centro-Oeste, Programa Cana IAC, Caixa Postal 66, 17201-970 Jaú (SP). marcelosilva@apta.sp.gov.br $\left({ }^{*}\right)$ Autor correspondente. 


\section{INTRODUÇÃO}

Novas variedades de cana-de-açúcar são obtidas anualmente nos programas de melhoramento existentes no Brasil. Uma variedade ideal é aquela que tem alta média de produção, mas um baixo grau de flutuação em seu desempenho quando cultivada sob diversas condições ambientais. O comportamento diferencial de genótipos em diferentes ambientes, isto é, interação genótipos por ambientes, resulta em alterações na classificação do genótipo em ensaios de competição ou mudanças nos valores entre as diferenças entre genótipos de um local para outro. A importância do estudo sobre interações genótipos $x$ ambientes em cana-de-açúcar é bem reconhecida (Milligan et al., 1990; Nahar e Khaleque, 2001; Rea e De Souza-Vieira, 2001, 2002; Kumar et al., 2004).

Portanto, uma nova variedade depende da comprovação de sua constância como material genético em grande amplitude de condições ambientais, denominado estabilidade fenotípica. $\mathrm{O}$ conhecimento dos parâmetros de estabilidade é uma ferramenta útil para separar diferenças genéticas ou ambientais entre variedades, pois apenas o valor da média de um determinado atributo é insuficiente para concluir sobre o comportamento do genótipo (KANG, 1998; RAIZER e VENCOVSKY, 1999).

Vários métodos estatísticos têm sido desenvolvidos e aplicados na análise de interação genótipo $\mathrm{x}$ ambiente e de estabilidade (FInLAY e WiLKINSON, 1963; EBERHART e RUSSELL, 1966; CROSSA, 1990; Vencovsky e Barriga, 1992; Ramalho et al., 1993; Cruz e RegazZI, 1994, Flores et al., 1996; Rosse et al., 2002). Esses métodos podem ser divididos em dois grandes grupos, modelos de regressão linear simples e bissegmentados (Lin et al., 1986). Segundo VencovsKy e BarRIGa (1992), os modelos lineares simples devem ser preferidos em relação aos bissegmentados sempre que o número de ambientes avaliados for menor que oito.

O método da regressão linear (Finlay e WiLKINSON, 1963; EbERHART e RuSSELL, 1966) é certamente o mais comumente usado para análise de estabilidade, devido a sua simplicidade e ao fato que sua informação sobre resposta adaptativa é facilmente aplicada aos ambientes (BECKER e LEÓN, 1988; ANNICCHIARICO, 1997). A interação genótipo $x$ ambiente da análise de variância é repartida dentro da heterogeneidade dos coeficientes de regressão $\left(\beta_{i}\right)$ e da soma dos desvios $\left(\Sigma \sigma_{d i}^{2}\right)$ das regressões. FinLAY e WiLKINSON (1963) definiram um genótipo com coeficiente de regressão igual a zero $\left(\beta_{i}=\right.$ 0) como estável, enquanto EBERHART e Russell (1966) definiram um genótipo com $\beta_{i}=1$, alto $\mathrm{R}^{2}$ e baixo $\sigma_{d i}^{2}$ ser estável. A maioria dos melhoristas considera $\sigma_{d i}^{2}$ como um parâmetro melhor que $\beta_{i}$ (BECKER e LEÓN, 1988).
De acordo com o modelo da regressão conjunta, um genótipo estável é aquele com alta produção média, $\beta_{i}=$ 1 e $\sigma_{d i}^{2}=0$ (EBERHART e RUSSELl, 1966).

Francis e Kannenberg (1978) usaram o coeficiente de variação $\left(\mathrm{cv}_{i}\right)$ e a média de rendimento de cada genótipo como uma medida da estabilidade em híbridos de milho. Um genótipo estável seria aquele que possui alta produção e um desempenho consistente nos diferentes ambientes. ReA e De SouZAVieira $(2001 ; 2002)$ utilizaram esse método em cana-de-açúcar como uma ferramenta suplementar na análise de estabilidade, com o diferencial de melhor visualizar grupos do que indivíduos estáveis.

Na Região Centro-Sul do Brasil, a cana-deaçúcar possui duas épocas de plantio, uma entre janeiro e março denominada cana de ano e meio, e outra chamada cana de ano, ou cana de doze meses, que se caracteriza pelo plantio realizado entre setembro e novembro, sendo a colheita realizada após 11 a 12 meses de idade (SiLva et al., 2001).

Segundo Landell e SiLVA (2004), a formação da produtividade de cana-de-açúcar, expressa em tonelada de cana por hectare (TCH), está em função dos atributos biométricos altura de colmos, número de perfilhos e diâmetro de colmos, os quais são determinantes para a expressão do potencial agrícola.

Os objetivos deste estudo foram avaliar o rendimento de doze genótipos plantados sob o sistema de cana de ano em três ambientes de produção, examinar a magnitude da interação genótipo $x$ ambiente e caracterizá-los usando parâmetros de estabilidade.

\section{MATERIAL E MÉTODOS}

Dez genótipos em fase experimental e duas variedades comerciais de cana-de-açúcar foram cultivados em ensaios repetidos em regiões produtoras contrastantes do Estado de São Paulo. Os genótipos foram: IAC87-3396, IAC91-1099, IAC91-3186, IAC915155, IACSP93-6006, IACSP94-2094, IACSP94-4004, IACSP94-6025, IACSP95-3018 e IACSP95-3028. Enquanto as variedades comerciais utilizadas como padrão foram SP80-1816 e RB72454. Todos os ensaios foram avaliados em três locais, Jaú, Piracicaba e São João da Boa Vista, nos ciclos de cana-planta e primeira soca em 2004 e 2005 respectivamente.

O delineamento experimental em cada local foi o de blocos ao acaso com quatro repetições. As parcelas consistiram de cinco sulcos de oito metros de comprimento, com espaçamento entre linhas de 1,5 m. Na tabela 1, são apresentadas informações mais detalhadas sobre os locais utilizados na experimentação. 
Tabela 1. Detalhes dos três locais de experimentação com doze genótipos de cana-de- açúcar no Estado de São Paulo

\begin{tabular}{|c|c|c|c|}
\hline \multirow{2}{*}{ Informações } & \multicolumn{3}{|c|}{ Locais } \\
\hline & Jaú & Piracicaba & São João da Boa Vista \\
\hline Data de plantio & $10 / 10 / 2003$ & $19 / 9 / 2003$ & $23 / 10 / 2003$ \\
\hline Data $1 .^{\mathrm{a}}$ colheita & $15 / 10 / 2004$ & $30 / 9 / 2004$ & $21 / 10 / 2004$ \\
\hline Ciclo plantio $-1^{\mathrm{a}} \mathrm{C}$ (dias) & 370 & 377 & 364 \\
\hline Data $2 .^{a}$ colheita & $03 / 11 / 2005$ & $04 / 10 / 2005$ & $18 / 10 / 2005$ \\
\hline Ciclo $1^{a} C-2^{a} C$ (dias) & 381 & 368 & 362 \\
\hline Latitude (Sul) & $22^{\circ} 17^{\prime}$ & $22^{\circ} 42^{\prime}$ & $21^{\circ} 55^{\prime}$ \\
\hline Longitude (Oeste) & $48^{\circ} 37^{\prime}$ & $47^{\circ} 38^{\prime}$ & $46^{\circ} 55^{\prime}$ \\
\hline Altitude $(\mathrm{m})$ & 580 & 546 & 688 \\
\hline Temperatura média máxima $\left({ }^{\circ} \mathrm{C}\right)$ & 28,9 & 27,8 & 28,0 \\
\hline Temperatura média mínima $\left({ }^{\circ} \mathrm{C}\right)$ & 16,6 & 14,3 & 12,1 \\
\hline Chuva total média annual (mm) & 1.344 & 1.257 & 1.500 \\
\hline Tipo de solo & LVaf & LBa & LVe \\
\hline
\end{tabular}

LVaf $=$ Latossolo Vermelho aluminoférrico; $\mathrm{LBa}=$ Latossolo bruno alumínico; LVe $=$ Latossolo Vermelho eutrófico $. \mathrm{C}=\mathrm{Colheita}$

Para a determinação dos atributos de produtividade e do conteúdo de sacarose (pol\% cana, PCC) foram coletados dez colmos seguidos na linha central da parcela. A medida de altura de colmos (A) foi feita da base até a inserção da folha +3 , utilizandose uma fita métrica. O diâmetro de colmos (D) foi medido por meio de um paquímetro na altura de $1 / 3$ do comprimento do colmo da base para a ponta (LANDell e Silva, 1995). Em seguida, os mesmos colmos foram enfeixados e levados para o laboratório para obtenção da PCC. O número de colmos (N) foi obtido pela contagem dos perfilhos da parcela após todas as amostragens, adicionando-se o número de 10 colmos retirados. A produtividade de colmos (tonelada de colmos por hectare, $\mathrm{TCH}$ ), dada pela massa de cana $\left(t \mathrm{th}^{-1}\right)$, foi feita no momento da colheita do ensaio, obtendo-se a massa das parcelas a partir de célula de carga, e adicionando-se a massa do feixe retirado durante a amostragem. A produtividade de açúcar (tonelada de pol por hectare, TPH) foi obtida em função do produto entre a TCH e a PCC correspondente de cada parcela.

Para a análise de variância, os dados foram agrupados por locais e analisados como um delineamento de blocos ao acaso. Para cada genótipo foi considerado um único valor de cada atributo, obtido a partir da média de duas colheitas. Todos os efeitos foram considerados fixos no modelo estatístico (NGUYen et al., 1980; TAI et al., 1982). As médias foram comparadas usando-se da diferença mínima significativa obtida pelo teste de Tukey.

A estabilidade fenotípica dos 12 genótipos em relação à $\mathrm{A}, \mathrm{D}, \mathrm{N}, \mathrm{TCH}, \mathrm{PCC}$ e TPH foi estimada com base no programa computacional Estabilidade
(2006). O modelo de regressão linear usado foi o de EBERHARt e RUSSELl (1966), conforme a seguinte expressão:

$$
Y_{i j}=\mu+\beta_{i} I_{j}+\delta_{i j}+{ }_{i j} \bar{\varepsilon}
$$

em que:

$\mathrm{Y}_{\mathrm{ij}}$ : é a média do genótipo $\mathrm{i}^{\mathrm{th}}$ no local $\mathrm{j}$;

$\mu$ : é a média geral do genótipo $i$;

$\beta_{i} I_{j}$ : é o coeficiente de regressão do genótipo $\mathrm{i}^{\text {th }}$ o qual mede a resposta do genótipo à variação do ambiente;

$I_{j}$ : é o índice ambiental, o qual é definido como a média do desvio de todos os genótipos em um dado local; local $j^{\text {th }}$;

$\delta_{i j}$ : é o desvio da regressão do genótipo $\mathrm{i}^{\text {th }}$ no

$\bar{\varepsilon}_{i j}$ : é a média do erro experimental.

Utilizou-se, também, o método de Francis e KANNENBERG (1978), que confronta o coeficiente de variação do genótipo com sua média. A análise consiste em representar esses valores em um gráfico, dividido por dois eixos, do coeficiente de variação médio e da média geral do atributo. grupos:

Dessa maneira, foram obtidos quatro

Grupo A: Alto valor do atributo e baixa variação;

Grupo B: Alto valor do atributo e grande variação;

Grupo C: Baixo valor do atributo e baixa variação;

Grupo D: Baixo valor do atributo e grande variação. 


\section{RESULTADOS E DISCUSSÃO}

Os resultados da análise de variância para altura de colmos, diâmetro de colmos, número de colmos por metro, $\mathrm{t} \mathrm{ha}^{-1}$ de colmos, pol\% cana e $\mathrm{t} \mathrm{ha}^{-1}$ de pol são apresentados na tabela 2. Efeito de genótipos e locais foi altamente significativo para todas as variáveis estudadas. A interação $G \times \mathrm{L}$ para número de colmos por metro, tonelada de colmos por hectare e tonelada de pol por hectare também foram altamente significativas. A interação $G \times L$ para diâmetro de colmos foi significativa a $5 \%$, enquanto não houve significância para a interação $G \times L$ quando estudada para as variáveis altura de colmos e pol\% cana. Os maiores valores de quadrados médios para local, em relação aos demais quadrados médios, indicam haver grande diferença no potencial produtivo entre os locais.

Tabela 2. Resultados da análise de variância para altura de colmos (A), diâmetro de colmos (D), número de colmos $(\mathrm{N})$, tonelada de colmos por hectare $(\mathrm{TCH})$, pol\% cana (PCC) e tonelada de pol por hectare (TPH) de 12 genótipos de cana-de-açúcar avaliados em três locais em sistema de plantio de cana de ano

\begin{tabular}{|c|c|c|c|c|c|c|c|}
\hline \multirow{2}{*}{ Fonte de variação } & \multirow{2}{*}{ GL } & \multicolumn{6}{|c|}{ Quadrados médios } \\
\hline & & A & $\mathrm{D}$ & $\mathrm{N}$ & $\mathrm{TCH}$ & PCC & $\mathrm{TPH}$ \\
\hline & & 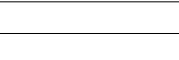 & - & unidade & t.ha ${ }^{-1}$ & $\%$ & t.ha ${ }^{-1}$ \\
\hline Bloco & 3 & $1181,66^{* *}$ & $0,03^{\mathrm{ns}}$ & $0,96^{\mathrm{ns}}$ & $20,72^{\mathrm{ns}}$ & $0,46^{\mathrm{ns}}$ & $1,81^{\mathrm{ns}}$ \\
\hline Genótipo (G) & 11 & $2293,16^{* *}$ & $0,38^{* *}$ & $6,23^{* *}$ & $1403,45^{* *}$ & $2,83^{* *}$ & $44,78^{* *}$ \\
\hline Local (L) & 2 & $56499,00 * *$ & $1,48^{* *}$ & $242,49^{* *}$ & $28084,69 * *$ & $35,56^{* *}$ & $1219,30^{* *}$ \\
\hline$G \times L$ & 22 & 183,86 & $0,03^{*}$ & $3,89^{* *}$ & $356,38^{* *}$ & 0,35 & $12,92^{* *}$ \\
\hline Resíduo & 105 & 202,75 & 0,01 & 1,05 & 71,37 & 0,28 & 2,28 \\
\hline Média & & 220,29 & 2,49 & 12,51 & 96,99 & 17,05 & 16,71 \\
\hline $\mathrm{CV}(\%)$ & & 6,46 & 4,68 & 8,20 & 8,71 & 3,12 & 9,04 \\
\hline
\end{tabular}

* $\mathrm{e}^{* *}=$ siginificante a $P \leq 0,05$ e $P \leq 0,01$ respectivamente. ns = não significativo.

Tais interações significativas são resultados das alterações no posicionamento do genótipo ou mudanças na magnitude das diferenças entre genótipos de um ambiente para outro. Por outro lado, os genótipos tiveram desempenho estável para altura de colmos e pol\% cana nos diferentes locais. Dessa forma, estando a produtividade de colmos em função dos atributos biométricos altura de colmos, número de perfilhos e diâmetro de colmos; a de açúcar em função do conteúdo de sacarose e da TCH (LANDELl e SILVA, 2004), infere-se que o maior responsável pelos efeitos significativos para TCH e TPH foi o número de colmos, seguido pelo diâmetro. Por essas observações, notam-se as dificuldades enfrentadas por melhoristas e fitotecnistas na recomendação do manejo varietal, as quais surgem, sobretudo, dos efeitos mascarados nos diversos ambientes. Assim, como relataram PhAm e KANg (1988), a interação genótipo $\mathrm{x}$ ambiente minimiza a confusão dos genótipos em relação ao desempenho de suas produções, sendo importante estudar em profundidade os níveis de vigor, o modelo de adaptação e a estabilidade dos genótipos em uma rede de experimentos.

As médias de dois cortes para altura de colmos, diâmetro de colmos e número de colmos por metro estão apresentadas na tabela 3. Altura de colmos variou de
192,25 a 239,21 cm, diâmetro de colmos de 2,03 a 2,66 $\mathrm{cm}$ e número de colmos por metro de 11,19 a 13,55. Os genótipos IAC87-3396, IAC91-1099, IAC91-3186, IAC91-5155, IACSP93-6006, IACSP95-3028, SP80-1816 e RB72454 foram significativamente $(P=0,05)$ superiores aos demais genótipos para altura de colmos. Para diâmetro de colmos, os genótipos com as maiores médias foram IAC91-1099, IACSP93-6006, IACSP94-6025, IACSP95-3018, IACSP95-3028 e RB72454. Para número de colmos por metro, os melhores desempenhos foram observados nos genótipos IAC87-3396, IAC91-1099, IACSP93-6006, IACSP94-2094, IACSP94-4004, IACSP94-6025, IACSP95-3018, IACSP95-3028 e SP80-1816.

$\mathrm{Na}$ tabela 4 são apresentadas as médias de duas colheitas de TCH, pol\% cana e TPH. A produtividade de colmos variou de 77,88 a 119,86 TCH, sendo os genótipos IAC91-1099 e IACSP94-4004 superiores aos demais $(P \leq 0,05)$. A variação de pol\% cana ficou entre 16,37 e $18,08 \%$, e os genótipos IACSP95-3018, IACSP95-3028 e SP80-1816 superaram siginificativamente o conteúdo de sacarose dos outros materiais. E finalmente, o menor valor de produtividade de açúcar foi de 13,05 e o maior de 20,72 TPH, em que os genótipos IAC91-1099 e IACSP94-4004 superaram o restante, inclusive os padrões SP80-1816 e RB72454. 
Tabela 3. Médias e parâmetros de estabilidade para altura de colmos, diâmetro de colmos, número de colmos de 12 genótipos de cana-de-açúcar avaliados em três locais em sistema de plantio de cana de ano

\begin{tabular}{|c|c|c|c|c|c|c|c|c|c|c|c|c|}
\hline \multirow{2}{*}{ Genótipo } & \multicolumn{4}{|c|}{ Altura de colmos $(\mathrm{cm})$} & \multicolumn{4}{|c|}{ Diâmetro de colmos $(\mathrm{cm})$} & \multicolumn{4}{|c|}{ Número de colmos } \\
\hline & Média & $\beta_{i}$ & $\delta_{i}$ & $\mathrm{CV}_{i}(\%)$ & Média & $\beta_{i}$ & $\delta_{i}$ & $\mathrm{CV}_{i}(\%)$ & Média & $\beta_{i}$ & $\delta_{i}$ & $\mathrm{CV}_{i}(\%)$ \\
\hline IAC $87-3396$ & 232,21 & $1,20 * *$ & 1,38 & 17,84 & 2,44 & 0,38 & $-2,45$ & 5,08 & 12,30 & $1,06^{* *}$ & 0,39 & 20,00 \\
\hline IAC91-1099 & 220,54 & $1,09^{* *}$ & 0,63 & 17,01 & 2,50 & $1,72^{* *}$ & 2,85 & 11,47 & 13,48 & $1,06^{* *}$ & 0,41 & 18,69 \\
\hline IAC91-3186 & 223,46 & $1,04^{* *}$ & 0,27 & 16,30 & 2,45 & $0,98^{*}$ & $-0,10$ & 6,70 & 11,84 & $1,18^{* *}$ & 1,09 & 22,43 \\
\hline IAC91-5155 & 239,21 & $1,00^{* *}$ & 0,03 & 14,40 & 2,28 & 0,11 & $-3,53$ & 2,42 & 11,19 & 0,56 & $-2,70$ & 11,33 \\
\hline IACSP93-6006 & 226,92 & $0,94^{* *}$ & $-0,41$ & 14,25 & 2,61 & 0,66 & $-1,35$ & 4,09 & 12,24 & $1,38^{* *}$ & 2,35 & 25,86 \\
\hline IACSP94-2094 & 192,25 & $0,91^{* *}$ & $-0,58$ & 16,35 & 2,03 & 0,90 & $-0,40$ & 7,93 & 13,51 & 0,79 ** & $-1,31$ & 19,10 \\
\hline IACSP94-4004 & 215,83 & $1,04^{* *}$ & 0,25 & 16,51 & 2,60 & $1,05^{* *}$ & 0,19 & 6,97 & 13,55 & $0,76^{* *}$ & $-1,49$ & 13,24 \\
\hline IACSP94-6025 & 197,75 & $1,22 * *$ & 1,53 & 21,59 & 2,66 & $1,22^{* *}$ & 0,89 & 8,18 & 12,52 & $1,09^{* *}$ & 0,55 & 19,84 \\
\hline IACSP95-3018 & 213,38 & $0,72^{* *}$ & $-1,90$ & 11,68 & 2,56 & $0,92^{*}$ & $-0,31$ & 6,01 & 12,25 & $0,77^{* *}$ & $-1,42$ & 14,24 \\
\hline IACSP95-3028 & 222,75 & $1,00^{* *}$ & $-0,03$ & 15,55 & 2,60 & $1,34^{* *}$ & 1,34 & 8,64 & 12,41 & $1,24^{* *}$ & 1,49 & 23,58 \\
\hline SP80-1816 & 231,42 & $0,88^{* *}$ & $-0,79$ & 13,45 & 2,38 & $1,26^{* *}$ & 1,03 & 9,25 & 12,80 & $1,06^{* *}$ & 0,37 & 18,64 \\
\hline RB72454 & 227,80 & $0,95^{* *}$ & $-0,37$ & 14,40 & 2,60 & $1,46^{* *}$ & 1,83 & 9,25 & 12,06 & $1,04^{* *}$ & 0,27 & 21,78 \\
\hline DMS (5\%) (Tukey) & 19,44 & - & - & - & 0,16 & - & - & - & 1,40 & - & - & - \\
\hline
\end{tabular}

* $\mathrm{e}^{* *}=$ significante a $P \leq 0,05$ e $P \leq 0,01$ respectivamente. $\beta i=$ coeficiente de regressão médio; $\delta i=$ desvio da regressão; $C V_{i}=$ coeficiente de variação do genótipo nos ambientes.

Os parâmetros de estabilidade para altura de colmos, diâmetro de colmos e número de colmos por metro são apresentados na tabela 3 , e os de $\mathrm{TCH}$, pol\% cana e TPH estão na tabela 4 . Adotando-se os critérios utilizados por EBERHART e Russell (1966), deve-se considerar para o julgamento da estabilidade de um genótipo nos ambientes, o seu comportamento médio, o componente linear $\left(\beta_{i}\right)$ e o componente não linear $\left(\sigma_{d i}^{2}\right)$. Um genótipo de ampla adaptabilidade foi definido como aquele com $\beta_{i}=1,0$ e alta estabilidade aquele com $\sigma_{d i}^{2}=0$.

Tabela 4. Médias e parâmetros de estabilidade para, produtividade de colmos (TCH), pol\% cana (PCC) e produtividade de açúcar (TPH) de 12 genótipos de cana-de-açúcar avaliados em três locais em sistema de plantio de cana de ano

\begin{tabular}{|c|c|c|c|c|c|c|c|c|c|c|c|c|}
\hline \multirow{2}{*}{ Genótipo } & \multicolumn{4}{|c|}{ Produtividade de colmos (t.ha $\left.{ }^{-1}\right)$} & \multicolumn{4}{|c|}{ Pol\% cana $(\%)$} & \multicolumn{4}{|c|}{ Produtividade de açúcar $\left(\right.$ t.ha $\left.^{-1}\right)$} \\
\hline & Média & $\beta_{i}$ & $\delta_{i}$ & $\mathrm{CV}_{i}(\%)$ & Média & $\beta_{i}$ & $\delta_{i}$ & $\mathrm{CV}_{i}(\%)$ & Média & $\beta_{i}$ & $\delta_{i}$ & $\mathrm{CV}_{i}(\%)$ \\
\hline IAC87-3396 & 97,50 & $1,00^{* *}$ & $-0,01$ & 24,83 & 17,00 & $0,97^{*}$ & $-0,12$ & 4,93 & 16,70 & $0,98^{* *}$ & $-0,17$ & 29,70 \\
\hline IAC91-1099 & 119,86 & $1,09^{* *}$ & 0,73 & 22,08 & 17,09 & $1,35^{* *}$ & 1,60 & 6,91 & 20,72 & $1,21^{* *}$ & 1,94 & 29,39 \\
\hline IAC91-3186 & 87,66 & $1,07^{* *}$ & 0,61 & 29,92 & 16,78 & $1,14^{* *}$ & 0,64 & 6,15 & 14,89 & $1,07^{* *}$ & 0,66 & 36,61 \\
\hline IAC91-5155 & 77,88 & 0,34 & $-5,34$ & 11,27 & 16,58 & 0,59 & $-1,86$ & 3,29 & 13,05 & 0,37 & $-5,91$ & 14,80 \\
\hline IACSP93-6006 & 95,15 & $1,38^{* *}$ & 3,07 & 35,06 & 17,05 & $1,33^{* *}$ & 1,53 & 6,86 & 16,48 & $1,37^{* *}$ & 3,50 & 41,95 \\
\hline IACSP94-2094 & 97,25 & 1,20 ** & 1,63 & 30,15 & 16,85 & $1,11^{* *}$ & 0,52 & 6,01 & 16,62 & $1,19 * *$ & 1,76 & 36,41 \\
\hline IACSP94-4004 & 112,41 & $1,05^{* *}$ & 0,41 & 22,93 & 17,20 & 0,77 & $-1,05$ & 4,09 & 19,43 & 1,00 ** & $-0,01$ & 26,05 \\
\hline IACSP94-6025 & 95,42 & $1,46^{* *}$ & 3,70 & 37,03 & 16,37 & $1,07^{* *}$ & 0,32 & 5,66 & 15,89 & $1,35^{* *}$ & 3,28 & 42,86 \\
\hline IACSP95-3018 & 93,17 & 0,24 & $-6,16$ & 6,45 & 17,67 & $0,95^{*}$ & $-0,25$ & 4,65 & 16,48 & 0,33 & $-6,32$ & 10,54 \\
\hline IACSP95-3028 & 91,76 & $1,05^{* *}$ & 0,44 & 27,90 & 18,08 & $0,97^{*}$ & $-0,11$ & 4,76 & 16,75 & $1,08^{* *}$ & 0,80 & 32,79 \\
\hline SP80-1816 & 94,46 & $1,00^{* *}$ & $-0,01$ & 25,64 & 17,39 & 0,75 & $-1,14$ & 4,06 & 16,50 & $0,95^{* *}$ & $-0,47$ & 29,03 \\
\hline RB72454 & 101,32 & $1,11^{* *}$ & 0,91 & 27,67 & 16,58 & $0,98^{*}$ & $-0,08$ & 5,16 & 17,01 & $1,10^{* *}$ & 0,96 & 33,62 \\
\hline DMS (5\%) (Tukey) & 11,53 & - & - & - & 0,73 & - & - & - & 2,06 & - & - & - \\
\hline
\end{tabular}

${ }^{*}$ e ${ }^{* *}=$ significante a $P \leq 0,05$ e $P \leq 0,01$ respectivamente. $\beta_{i}=$ coeficiente de regressão médio; $\delta_{i}=$ desvio da regressão; $C V_{i}=$ coeficiente de variação do genótipo nos ambientes. 
Neste estudo, os valores de coeficiente de regressão variaram de 0,72 (IACSP95-3018) a 1,22 (IACSP94-6025) para altura de colmos; de 0,11 (IAC91-5155) a 1,72 (IAC91-1099) para diâmetro de colmos; de 0,56 (IAC91-5155) a 1,38 (IACSP93-6006) para número de colmos por metro; de 0,24 (IACSP953018) a 1,38 (IACSP93-6006) para $\mathrm{TCH}$; de 0,59 (IAC91-5155) a 1,35 (IAC91-1099) para pol\% cana, e de 0,33 (IACSP95-3018) a 1,37 (IACSP93-6006) para TPH. Grandes variações no coeficiente de regressão revelam que os genótipos foram diferentemente responsivos aos ambientes. Pfahler e LinsKen (1979) demonstraram que a grande utilidade do coeficiente de regressão é identificar a resposta de determinado parâmetro à variabilidade entre os ambientes.

Em termos de adaptação geral, ou seja, coeficiente de regressão $\beta_{i}$ ao redor da unidade e com rendimento próximo da média do componente de produção, os seguintes genótipos poderiam ser classificados: IAC91-1099, IAC91-3186, IAC91-5155, IACSP93-6006, IACSP94-4004, IACSP95-3028 e RB72454 para altura de colmos; IAC91-3186 e IACSP94-4004 para diâmetro de colmos; IAC87-3396, IAC91-1099, IACSP946025 e SP80-1816 para número de colmos por metro; IAC87-3396, IAC91-1099, IACSP94-4004, IACSP95-3028, SP80-1816 e RB72454 para TCH; IAC87-3396, IACSP942094, IACSP95-3018 e IACSP95-3028 para pol\% cana, e IAC87-3396, IACSP94-4004, IACSP95-3028, SP80-1816 e RB72454 para TPH.

Quanto à adaptação específica a ambientes de altos rendimentos, seriam agrupados aqueles genótipos com coeficiente de regressão maior que a unidade e alto valor do componente de produção. Para altura de colmos, tem-se apenas o IAC87-3396; para diâmetro de colmos tem-se IAC91-1099, IACSP94-6025, IACSP953028 e RB72454; para número de colmos por metro, $\mathrm{TCH}$ e pol\% cana nenhum genótipo pôde ser considerado, e para TPH apenas o IAC91-1099.

Outra classificação seria a de adaptação específica a ambientes de baixos rendimentos, onde se agrupariam genótipos de alto valor do componente de produção, mas com coeficiente de regressão menor que a unidade. Neste caso, formaria o grupo para altura de colmos apenas SP80-1816; para diâmetro de colmos, apenas IACSP93-6006; para número de colmos por metro, IACSP94-2094 e IACSP94-4004; para $\mathrm{TCH}$ e TPH, nenhum genótipo foi considerado, e para pol\% cana os genótipos IACSP94-4004 e SP80-1816 se qualificaram nesse grupo.

Finalmente, a última classificação seria a de adaptação pobre a todos ambientes, e nesse grupo estariam todos os genótipos com coeficiente de regressão menor que um, com desvio da regressão distante de zero e baixos rendimentos.
FRANCIS e KANNENBERG (1978) propuseram um método descritivo o qual contrapõe-se em um gráfico a média do componente de produção com o seu coeficiente de variação. O gráfico, então, é dividido pela média do coeficiente de variação e pela média do componente de produção formando quatro quadrantes, os quais são classificados nos seguintes grupos: Grupo A, alto rendimento e pouca variação; Grupo B, alto rendimento e alta variação; Grupo C, baixo rendimento e pouca variação, e Grupo D, baixo rendimento e alta variação. Um genótipo estável é o que possui alto rendimento e desempenho consistente através dos ambientes, portanto, são aqueles localizados no quadrante A.

Dessa forma, para altura de colmos seriam agrupados como estáveis os genótipos IAC91-5155, IACSP93-6006, IACSP95-3028, SP80-1816 e RB72454 (Figura 1). Para diâmetro de colmos o grupo dos estáveis seria formado por IACSP93-6006, IACSP944004 e IACSP95-3018 (Figura 2). Estariam no grupo A, para número de colmos por metro, os genótipos IAC91-1099, IACSP94-4004 e SP80-1816 (Figura 3). Os genótipos estáveis para TCH seriam IAC87-3396, IAC91-1099 e IACSP94-4004 (Figura 4). Para pol\% cana, estariam agrupados como estáveis IACSP944004, IACSP95-3018, IACSP95-3028 e SP80-1816 (Figura 5). E, por fim, para TPH, os genótipos estáveis seriam IAC91-1099 e IACSP94-4004 (Figura 6).

Com base nos dois métodos de análise, os genótipos mais estáveis foram IAC91-5155, IACSP936006, IACSP95-3028 e RB72454 para altura de colmos; IACSP94-4004 para diâmetro de colmos; IAC91-1099 e SP80-1816 para número de colmos por metro; IAC873396, IAC91-1099 e IACSP94-4004 para TCH; IACSP95-3018, IACSP95-3028 e SP80-1816 para pol\% cana, e IACSP94-4004 para TPH, mostrando ampla adaptabilidade às condições de plantio sob o sistema cana de ano nessas três regiões. Essas informações também sugerem que esses métodos podem ser usados como uma ferramenta para auxiliar na recomendação do manejo desses genótipos em plantio como cana de ano quando interações genótipo $\mathrm{x}$ ambiente estão presentes. Rea e De SouZA-VieIRa $(2001 ; 2002)$ também relataram que o método do coeficiente de variação pode ser usado de forma adicional ao método de regressão linear no processo de melhoramento, seleção e recomendação de variedades.

Esses genótipos também podem ser considerados em futuros programas de cruzamentos a fim de incorporar estabilidade a esses componentes para esse sistema de produção, pois conforme SINGH e Gupta (1988) é possível que genótipos estáveis carreguem genes para estabilidade, úteis em programas de melhoramento, por produzirem variedades que elevarão a produtividade com baixo custo de produção. 


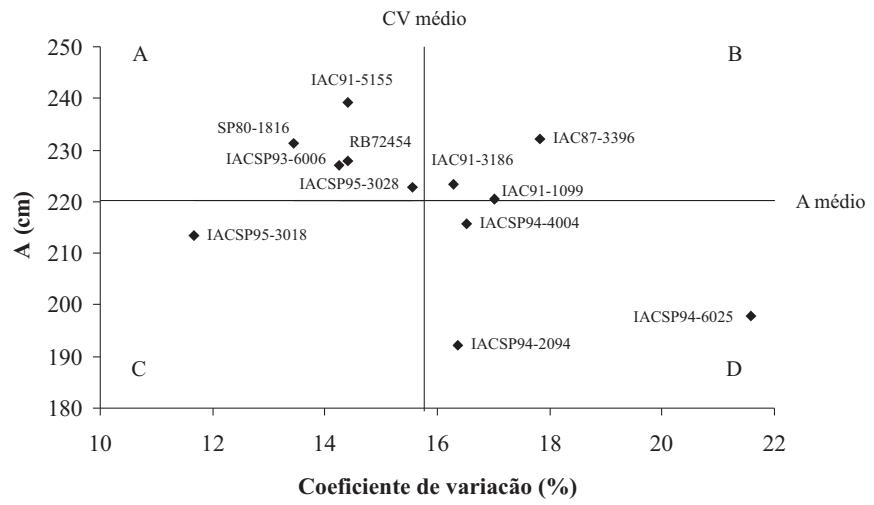

Figura 1. Média de altura de colmos (A) por coeficiente de variação $(\mathrm{CV})$ para 12 genótipos de cana-de-açúcar em três ambientes no Estado de São Paulo (Jaú, Piracicaba e São João da Boa Vista).

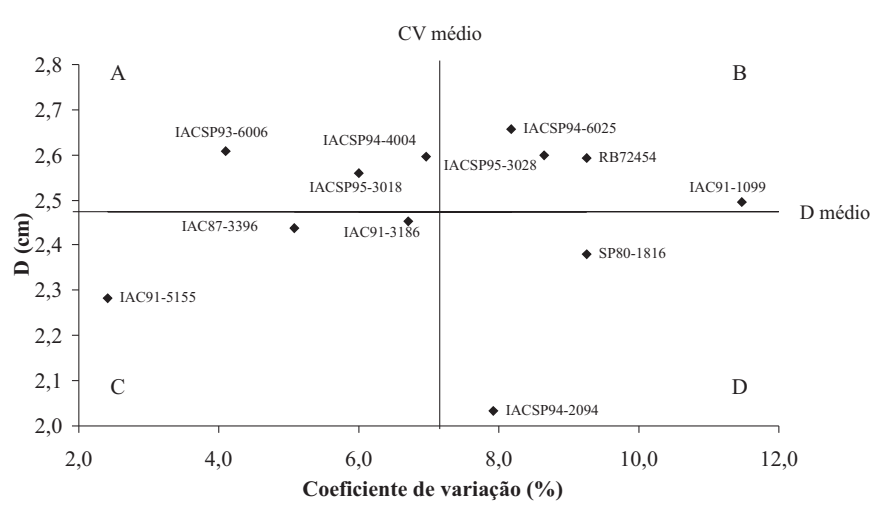

Figura 2. Média de diâmetro de colmos (D) por coeficiente de variação $(\mathrm{CV})$ para 12 genótipos de cana-de-açúcar em três ambientes no Estado de São Paulo (Jaú, Piracicaba e São João da Boa Vista).

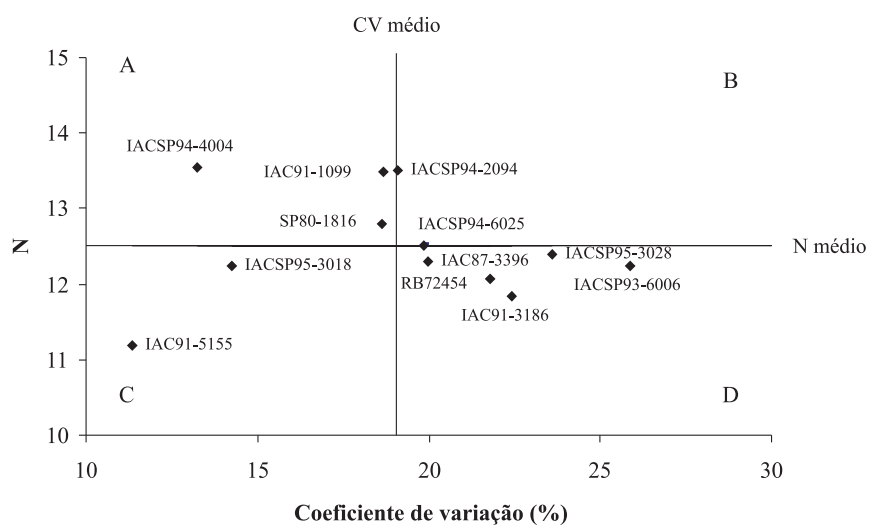

Figura 3. Média de número de colmos por metro $(\mathrm{N})$ por coeficiente de variação $(\mathrm{CV})$ para 12 genótipos de canade-açúcar em três ambientes no Estado de São Paulo (Jaú, Piracicaba e São João da Boa Vista).

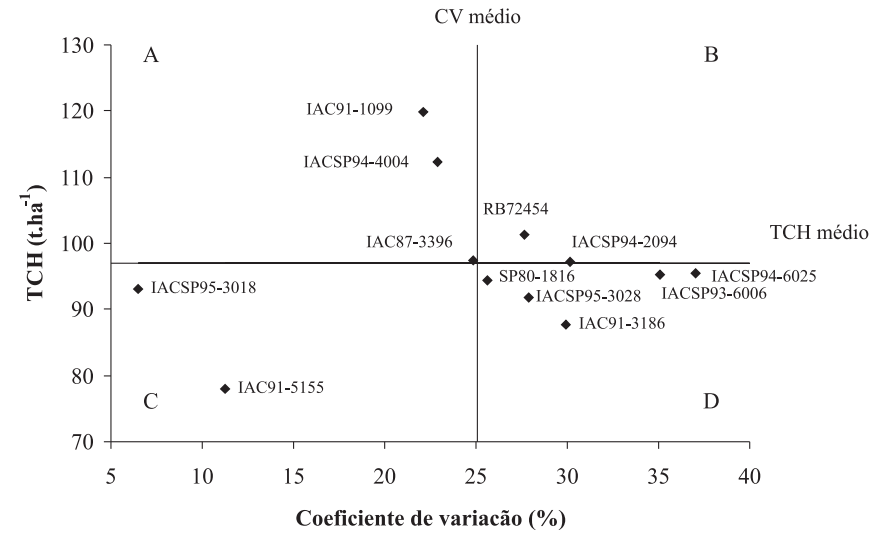

Figura 4. Média de tonelada de colmos por hectare $(\mathrm{TCH})$ por coeficiente de variação $(\mathrm{CV})$ para 12 genótipos de cana-de-açúcar em três ambientes no Estado de São Paulo (Jaú, Piracicaba e São João da Boa Vista).

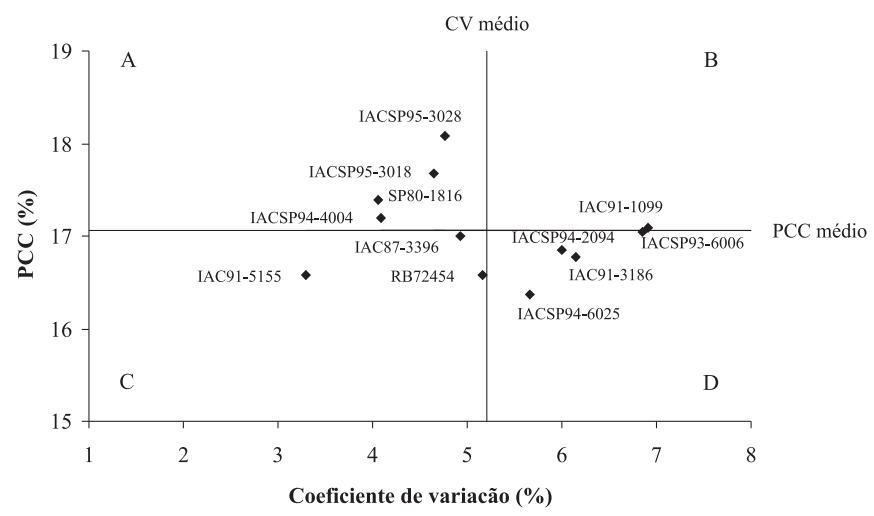

Figura 5. Média de pol\% cana (PCC) por coeficiente de variação $(\mathrm{CV})$ para 12 genótipos de cana-de-açúcar em três ambientes no Estado de São Paulo (Jaú, Piracicaba e São João da Boa Vista).

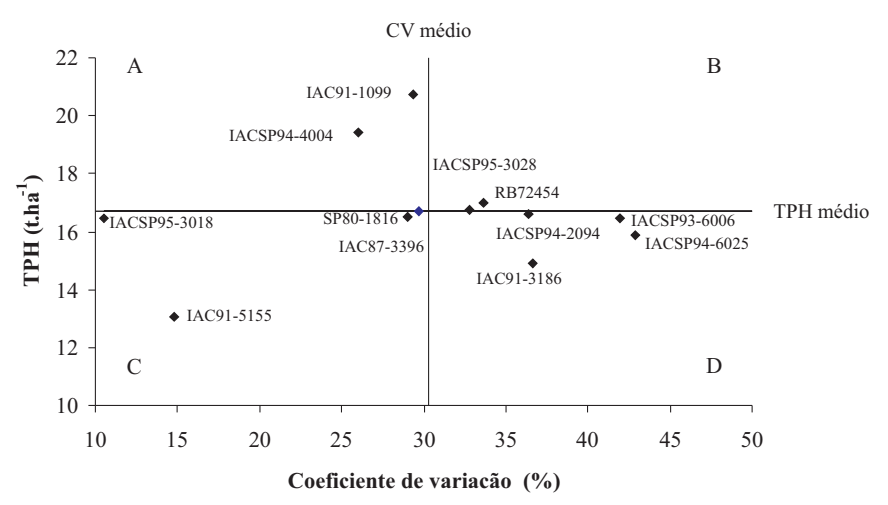

Figura 6. Média de tonelada de pol por hectare (TPH) por coeficiente de variação $(\mathrm{CV})$ para 12 genótipos de canade-açúcar em três ambientes no Estado de São Paulo (Jaú, Piracicaba e São João da Boa Vista). 


\section{CONCLUSÕES}

1. Número de colmos por metro é o atributo biométrico mais afetado pela interação genótipo $x$ ambiente.

2. A interação significativa genótipo $x$ ambiente para diâmetro de colmos, número de colmos por metro, TCH e TPH e a alteração dos genótipos dentro de cada atributo sugerem que estratégias específicas devem ser adotadas para o melhoramento e manejo de variedades para o sistema cana de ano.

3. Os genótipos IAC87-3396, IAC91-1099 e IACSP4004 podem ser recomendados como opções de plantio sob sistema cana de ano nas regiões de Jaú, Piracicaba e São João da Boa Vista.

4. Novos genótipos liberados para plantio comercial devem conter informações de interação genótipo $\mathrm{x}$ ambiente e de estabilidade, inclusive com indicação de sua adaptação específica e/ou geral.

\section{AGRADECIMENTOS}

Ao Dr. Marcos G.Andrade Landell, do Centro de Cana IAC, pela disponibilidade dos genótipos IAC. A Dra. Raffaella Rossetto, da APTA Centro-Sul, e aos diretores da Usina São João Dedini, pelo fornecimento da área e pela assistência de campo.

\section{REFERÊNCIAS}

ANNICCHIARICO, P. Joint regression vs. AMMI analysis of genotype-environment interactions for cereals in Italy. Euphytica, Dordrecht, v. 94, n. 1, p. 53-62, 1997.

BECKER, H.C.; LEÓN, J. Stability analysis in plant breeding. Plant Breeding, Hamburg, v. 101, p. 1-23, 1988.

CROSSA, J. Statistical analyses of multi-location trials. Advances in Agronomy, San Diego, v.44, p. 55-85, 1990.

CRUZ, C.D.; REGAZZI, A.J. Modelos biométricos aplicados ao melhoramento genético. Viçosa: Universidade Federal de Viçosa, 1994. 390p.

EBERHART, S.A.; RUSSELL, W.A. Stability parameters for comparing varieties. Crop Science, Madison, v. 6, n. 1, p.3640, 1966.

ESTABILIDADE. Programa computacional. Disponível em: http://www.dex.ufla.br/ danielff/softwares.htm.> Acesso em 7 de nov. de 2006.

FINLAY, K.W.; WILKINSON, G.N. The analysis of adaptation in a plant-breeding programme. Australian Journal of Agricultural Research, Melbourne, v. 14, p. 742-752, 1963.
FLORES, F.M., MORENO, T.; CUBERO, J.I. A comparison of univariate and multivariate methods to analyse $\mathrm{G} \times \mathrm{E}$ interaction. Field Crops Research, Amsterdam, v. 47, n. 2/3, p. 117-127, 1996.

FRANCIS, T.R.; KANNENBERG, L.W. Yield stability studies in short-season maize. I. A descriptive method for grouping genotypes. Canadian Journal of Plant Science, Otawa, v. 58, p. 1029-1034, 1978.

KANG, M.S. Using genotype-by-environment interaction for crop cultivar development. Advances in Agronomy, San Diego, v. 62, p.199-253, 1998.

KUMAR, S.; SINGH, P.K.; SINGH, J.; SWAPNA, M. Genotypes x environment interaction analysis for quantitative traits in sugarcane. Indian Sugar, New Delhi, v. 53, n. 10, p.813-818, 2004.

LANDELL, M.G.A.; SILVA, M.A. Manual do experimentador: melhoramento da cana-de-açúcar. In: Metodologia de Experimentação: ensaios de competição em cana-de-açúcar. Pindorama: Instituto Agronômico, 1995. Não paginado. (Apostila de Treinamento Interno)

LANDELL, M.G.A.; SILVA, M.A. As estratégias de seleção da cana em desenvolvimento no Brasil. Visão Agrícola, Piracicaba, n. 1, p.18-23, 2004.

LIN, C.S.; BINNS, M.R.; LEFKOVITCH, L.P.Stability analysis: where do we stand? Crop Science, Madison, v. 26, n. 4, p.894-900, 1986.

MILLIGAN, S.B.; GRAVOIS, K.A.; BIRCHOFF, K.P.; MARTIN, F.A. Crop effects on broad-sense heritabilities and genetic variances of sugarcane yield components. Crop Science, Madison, v. 30, p. 344-349, 1990.

NAHAR, S.M.N.; KHALEQUE, M.A. Genotype - environment interaction and stability in sugarcane. Indian Sugar, New Delhi, v. 50, n. 11, p.811-820, 2001.

NGUYEN, H.T.; SLEPER, D.A.; HUNT, K. Genotype $x$ environment interactions and stability analysis for herbage yield of tall fescue synthetics. Crop Science, Madison, v. 20, p.221-224, 1980.

PFAHLER, P.L.; LINSKENS, H.F. Yield stability and population diversity in oats (Avena sp.). Theoretical and Applied Genetics, Berlin, v.54, p.1-5, 1979.

PHAM, H.N.; KANG, M.S. Interrelationships among respectability of several stability statistics estimated from international maize trials. Crop Science, Madison, v.28, p. 925-928, 1988.

RAIZER, A.J.; VENCOVSKY, R. Estabilidade fenotípica de novas variedades de cana-de-açúcar para o Estado deSão Paulo. Pesquisa Agropecuária Brasileira, Brasília, v.34, n.12, p. 2241-2246, 1999.

RAMALHO, M.A.P.; SANTOS, J.B.; ZIMMERMANN, M.J.O. Genética quantitativa em plantas autógamas: aplicações no melhoramento do feijoeiro. Goiânia: UFG, 1993. 271p.

ROSSE, L.N.; VENCOVSKY, R.; FERREIRA, D.F. Comparação de métodos de regressão para avaliar a estabilidade fenotípica em cana-de-açúcar. Pesquisa Agropecuária Brasileira, Brasília, v.37, n.1, p. 25-32, 2002. 
REA, R.; SOUSA-VIEIRA, O. Interaccion genotipo $x$ ambiente $y$ analisis de estabilidade en ensayos regionales de caña de azúcar en Venezuela. Caña de Azúcar, Yaracuy, v.19, n.(único), p.3-15, 2001.

REA, R.; SOUSA-VIEIRA, O. Genotype $x$ enviroment interactions in sugarcane yield trials in the Central-Western region of Venezuela. Interciencia, Caracas, v.27, n.11, p.620624, 2002.

SILVA, M. A.; LANDELL, M. G. de A.; CAMPANA, M. P. Novas opções varietais IAC para plantio no sistema de cana-de-ano. STAB - Açúcar, Álcool e Subprodutos, Piracicaba, v. 19, n. 3, p. 43-45, 2001.
SINGH, S.V.; GUPTA, P.K. Phenothypic stability for tuber yield potato (Solanum tuberosum L.). Indian Journal of Genetics and Plant Breeding, v.48, p.1223-1232, 1988.

TAI, P.Y.P.; RICE, E.R.; CHEW, V.; MILLER, J.D. Phenotypic stability analyses of sugarcane cultivar performance tests. Crop Science, Madison, v. 22, p. 1179-1184, 1982.

VENCOVSKY, R.; BARRIGA, P. Genética biométrica no fitomelhoramento. Ribeirão Preto: Sociedade Brasileira de Genética, 1992. 486p. 